\title{
ABUSO SEXUAL NA INFÂNCIA E ADOLESCÊNCIA: PERFIL DAS VÍTIMAS E AGRESSORES EM MUNICÍPIO DO SUL DO BRASIL ${ }^{1}$
}

\author{
Christine Baccarat de Godoy Martins², Maria Helena Prado de Mello Jorge ${ }^{3}$
}

\footnotetext{
${ }^{1}$ Artigo extraído da tese intitulada - Violência contra menores de 15 anos: análise epidemiológica de suas notificações. Faculdade de Saúde Pública da Universidade de São Paulo (USP), 2008.

${ }^{2}$ Doutora em Saúde Pública. Docente da Universidade Federal de Mato Grosso. Mato Grosso, Brasil. E-mail: leocris2001@terra. com.br

${ }^{3}$ Doutora em Saúde Pública. Professor Associado da Faculdade de Saúde Pública da USP. São Paulo, Brasil. E-mail: mhpjorge@ usp.br
}

RESUMO: Estudo descritivo e de corte transversal que objetivou conhecer as características do abuso sexual em crianças e adolescentes de zero a 14 anos, a partir dos casos registrados nos Conselhos Tutelares e programas de atendimento do município de LondrinaPR, em 2006. Os dados foram coletados por meio de formulário e posteriormente analisados por frequência (absoluta e relativa) e proporção. Dos 186 casos, as vítimas foram predominantemente do sexo feminino $(74,2 \%)$ e o risco de incidência foi maior na idade de 10 anos entre as meninas (coeficiente de cinco por 1.000); 97,3\% dos agressores eram do sexo masculino; maior parte dos abusos ocorreu na residência das vítimas (52,7\%) e durou menos de seis meses (57\%). Houve lesão corporal em $90,3 \%$ dos casos, com seqüela física e psicológica em $97,8 \%$. O abuso sexual entre crianças e adolescentes constitui-se um problema de saúde pública, além da estreita interface com as questões policiais e jurídicas.

DESCRITORES: Maus-tratos sexuais infantis. Violência sexual. Criança. Adolescente.

\section{CHILDHOOD AND ADOLESCENT SEXUAL ABUSE: PROFILE OF THE VICTIMS AND AGGRESSORS IN A COUNTY IN THE SOUTH OF BRAZIL}

\begin{abstract}
This descriptive and transversal study aims to better understand the characteristics of childhood and adolescent (up to 14 years of age) sexual abuse through analyzing cases registered in Tutelary Councils and care programs in Londrina, Paraná, Brazil in 2006. Data was collected from the records using a questionnaire and later analyzed for absolute and relative frequency and proportion. Of the 186 cases studied, the victims were predominantly female $(74.2 \%)$ and the highest risk of incidence was at age 10 for girls (incidence coefficient 5 of 1000). Among aggressors, $97.3 \%$ were male; with the majority of abuses (52.7\%) occurring in the victim's house and lasting for a period of less than 6 months (57.0\%). Physical harm occurred in $90.3 \%$ of the victims and $97.8 \%$ presented physical and psychological sequelae. Childhood and adolescent sexual abuse constitutes a significant public health problem, besides the direct interface with police and judicial questions.
\end{abstract}

DESCRIPTORS: Sexual child, abuse. Sexual violence. Child. Adolescent.

\section{ABUSO SEXUAL EN LA INFANCIA Y LA ADOLESCENCIA: EL PERFIL DE LAS VÍCTIMAS Y DE SUS AGRESORES EN UNA CIUDAD DEL SUR DE BRASIL}

RESUMEN: Este estudio descriptivo y trasversal tuvo como objetivo conocer las características del abuso sexual en niños y adolescentes, desde el nacimiento hasta los catorce años, de los casos registrados en los Consejos Tutelares y en programas de atención en la ciudad de Londrina-PR, en 2006. Los datos se recolectaron mediante un cuestionario y después se analizaron por la frecuencia (absoluta y relativa) y la proporción. De los 186 casos estudiados, las víctimas eran en su mayoría mujeres (74,2\%), y el riesgo de incidencia fue mayor a la edad de 10 años en las niñas (coeficiente de 5 por 1.000); el (97,3\%) de los autores eran hombres. La mayoría de los abusos ocurrieron en la residencia de las víctimas (52,7\%) y duró menos de seis meses (57\%). Hubo lesión corporal en 90,3\% de las víctimas, con secuelas físicas y psicológicas en el $97,8 \%$. El abuso sexual en niños y adolescentes se constituye en un problema de salud publica, además de la interfaz con las cuestiones policiales y judiciales.

DESCRIPTORS: Abuso sexual infantil. Violencia sexual. Niño. Adolescente. 


\section{INTRODUÇÃO}

Os abusos sexuais, que vão desde tentativas de atentado ao pudor até o estupro, ${ }^{1}$ constituem, atualmente, importante evento mórbido que vitimiza crianças e adolescentes ${ }^{2-4}$ adquirindo caráter endêmico e convertendo-se em um complexo problema de saúde pública, ${ }^{5-6}$ caracterizando uma das formas mais graves de violação dos direitos humanos $^{7}$ e ocasionando impactos relevantes na saúde física e mental de suas vítimas. ${ }^{5}$

Reconhecendo a importância deste agravo e objetivando traçar a conduta frente ao abuso sexual, o Ministério da Saúde, em 1999, através da Secretaria de Políticas de Saúde, normatizou a prevenção e tratamento dos agravos resultantes da violência sexual contra mulheres e adolescentes ${ }^{8}$ e em 2001, o Ministério da Justiça, pela Secretaria de Estado dos Direitos Humanos, traçou um Plano Nacional de Enfrentamento da Violência Sexual Infanto-Juvenil. ${ }^{9}$

Estatísticas apontam que, na Costa Rica, 3,2\% das meninas e $13,0 \%$ dos meninos são abusados sexualmente ao ano. Na Nicarágua, estes percentuais são de $26,0 \%$ para as meninas e $20,0 \%$ para os meninos. ${ }^{10-11} \mathrm{Na}$ Venezuela, uma investigação documental revelou que, a cada 100 vítimas de violência sexual, 20 são menores de seis anos. ${ }^{12}$ Estudo de prevalência e incidência de abuso infantil, ao comparar dados nos diferentes países, aponta a negligência, o abuso físico e o sexual entre os mais freqüentes na população infantil. ${ }^{13}$ Pesquisa domiciliar realizada na Carolina do Norte e do Sul evidenciou uma incidência de 23 casos de violência sexual por 1.000 crianças (29 por 1.000 entre as meninas e três por 1.000 entre os meninos). ${ }^{4}$ Nos Estados Unidos, a incidência de abuso sexual já chegou a 2,6 por 1.000 adolescentes na faixa etária de 12 a 14 anos e de 2,9 por 1.000 adolescentes de 15 a 19 anos de idade. ${ }^{14}$

Em São Paulo, os serviços de atendimento apontam que grande parte dos casos de violência doméstica contra crianças e adolescentes são por abuso sexual. ${ }^{15}$ Em Curitiba, os serviços que compõem a Rede de Proteção à Criança e ao Adolescente em Situação de Risco para a Violência verificou que a violência sexual representa 6,2\% das notificações. ${ }^{16}$

Entretanto, o abuso sexual, apesar de sua crescente incidência e da relevância do diagnóstico para a identificação precoce de fatores de risco e para a implantação de programas preventivos, ${ }^{17}$ ainda constitui-se o tipo de violência contra crianças e adolescentes de menor notificação, envolvido num profundo pacto de silêncio e tabus culturais, o que contribui para o desconhecimento de sua real incidência e mecanismos. ${ }^{18}$ Desta forma, estudos localizados podem contribuir para que se conheça melhor a ocorrência deste evento na população infanto-juvenil. ${ }^{19}$

Neste sentido, o presente estudo teve o objetivo de descrever as características do abuso sexual de crianças e adolescentes de zero a 14 anos de idade, quanto às características do agressor, da vítima e circunstâncias da violência, a partir dos casos notificados no município de Londrina-PR, no ano de 2006, uma vez que ainda não há um perfil epidemiológico deste evento no município, fornecendo subsídios para propostas de intervenção e prevenção.

\section{METODOLOGIA}

Trata-se de estudo descritivo, de corte transversal, de crianças e adolescentes de zero a 14 anos, vítimas de abuso sexual, atendidos pelos Conselhos Tutelares de Londrina do município (Conselho Tutelar Centro, Conselho Tutelar Norte e Conselho Tutelar Sul) e pelos programas e projetos de atendimento a crianças e adolescentes vitimizados (Programa Sentinela da Prefeitura Municipal e projeto de extensão da Universidade Estadual de Londrina - De Olho no Futuro), incluindo, portanto, todos os casos notificados no município durante o ano de 2006.

O Programa Sentinela, implantado em 14 de fevereiro de 2002, é uma ação integrada entre Secretaria Municipal de Saúde, Secretaria Municipal de Assistência Social, Secretaria Municipal da Educação eSecretaria Municipal da Mulher e através de parceria com o Núcleo Social Evangélico de Londrina (NUSELON), cujo objetivo é propiciar atendimento a crianças e adolescentes vítimas de violência sexual, buscando fortalecer a auto-estima da vítima por meio de atendimento assistencial e psicológico (individual, em grupo e familiar), tendo como referência os eixos estratégicos contemplados no Plano Nacional de Enfrentamento à Violência Sexual Infanto-Juvenil. ${ }^{20}$

O projeto De Olho no Futuro (projeto de ação interdisciplinar no combate à violência praticada contra crianças e adolescentes, aprovado pelo $\mathrm{CNPq}$ ) é desenvolvido pelos Departamentos de Direito, Departamento de Serviço Social e De- 
partamento de Psicologia Social da Universidade Estadual de Londrina, integrando ensino, pesquisa e extensão, atendendo crianças e adolescentes vítimas de violência encaminhados pelos Conselhos Tutelares, Promotoria da Vara da Infância e da Juventude, pelo Hospital Universitário de Londrina da Universidade Estadual de Londrina (UEL), tendo por objetivo "estudar e levantar coletivamente os procedimentos necessários para intervenção do problema, sem perder de vista a especificidade de cada área de atuação". ${ }^{21: 25}$

Foram considerados critérios de inclusão crianças e adolescentes entre zero e 14 anos, residentes em Londrina, vítimas de abuso sexual, cuja notificação se deu no ano de 2006.

As categorias de análise foram: quem denunciou o abuso, o sexo e a idade das vítimas, o sexo e a idade dos agressores, o vínculo entre agressores e vítimas, a situação de vulnerabilidade associada ao agressor (como alcoolismo, drogadição, desemprego, etc.), o local onde o mesmo ocorreu, a freqüência (quantas vezes) e a duração do abuso, a lesão ocasionada e o segmento corpóreo afetado, a necessidade de internação e as sequelas decorrentes da violência.

A fonte de dados foram os prontuários das instituições e para a coleta utilizou-se formulário com questões fechadas, previamente testado. Os dados foram coletados no ano de 2007, por uma equipe de 30 alunos do Curso de Enfermagem da UEL, rigorosamente treinados e supervisionados pela pesquisadora.

Foi considerado, na coleta de dados, o número de notificações de violência sexual e não o número de crianças e adolescentes, considerando que cada um deles possa sofrer violência em momentos e circunstâncias diferentes. Para os casos com mais de uma notificação referente ao mesmo ato violento, a mesma data da violência e mesma circunstância, as informações foram complementadas em uma única ficha e excluídas as demais a fim de evitar a duplicidade de dados. Para os casos em que as notificações correspondiam a eventos diferentes (praticados em datas diferentes, pelo mesmo agressor ou não), foram mantidos os dois registros.
Os dados foram processados eletronicamente pelo programa computacional EpiInfo $6.04 \mathrm{~d},{ }^{22}$ sendo analisados quantitativamente, em freqüência (relativa e absoluta) e proporção.

O projeto de pesquisa foi submetido à apreciação do Comitê de Ética em Pesquisa da Faculdade de Saúde Pública da Universidade de São Paulo, sendo aprovado em outubro de 2006 sob o protocolo n $315 / 2006$.

Os dados coletados nas fichas de notificação dos Conselhos Tutelares preservaram o anonimato das pessoas envolvidas e o estudo está em conformidade com a resolução $n^{\circ}$ 196/96 do Conselho Nacional de Saúde.

\section{RESULTADOS}

Foram notificados 186 casos de abuso sexual contra crianças e adolescentes (de zero a 14 anos) no município de Londrina-Pr, no ano de 2006. Os principais denunciantes foram familiares como tios, cunhados, irmãos e primos $(67,2 \%)$, seguidos pela mãe $(8,1 \%)$, diretor da escola $(7,0 \%)$, pai $(5,4 \%)$, profissionais de saúde $(3,2 \%)$, vizinhos $(2,7 \%)$, a própria vítima $(2,7 \%)$, avós $(1,6 \%)$, anônimos $(1,1 \%)$ e outros $(1,1 \%)$.

Entre as vítimas, houve predomínio do sexo feminino (74,2\%), numa razão de masculinidade de 0,3 ou, ainda, numa proporção de 2,9 meninas para cada menino. Entre as meninas, observou-se concentração de casos na faixa etária dos 10 aos 14 anos $(58,0 \%)$, seguida pela faixa etária de cinco a nove $(31,8 \%)$ e de zero a quatro anos (10,1\%). Entre os meninos, a faixa etária com maior frequiência de casos foi de cinco a nove anos $(66,7 \%)$, seguida pela faixa de 10 a $14(25,0 \%)$ e de zero a quatro anos $(8,4 \%)$.

Calculando-se os coeficientes de incidência com base populacional (considerando no denominador a população residente no município em cada faixa etária, segundo o sexo, no mesmo ano), observou-se maior risco nas idades de 10 anos para as meninas $(5,0$ por 1.000$)$ e de nove anos para os meninos (2,3 por 1.000). Nota-se também o aumento do risco entre as meninas após os oito anos de idade, ao contrário dos meninos em que os coeficientes declinam a partir desta idade. O coeficiente total foi de 1,4 por 1.000 crianças (Figura 1). 


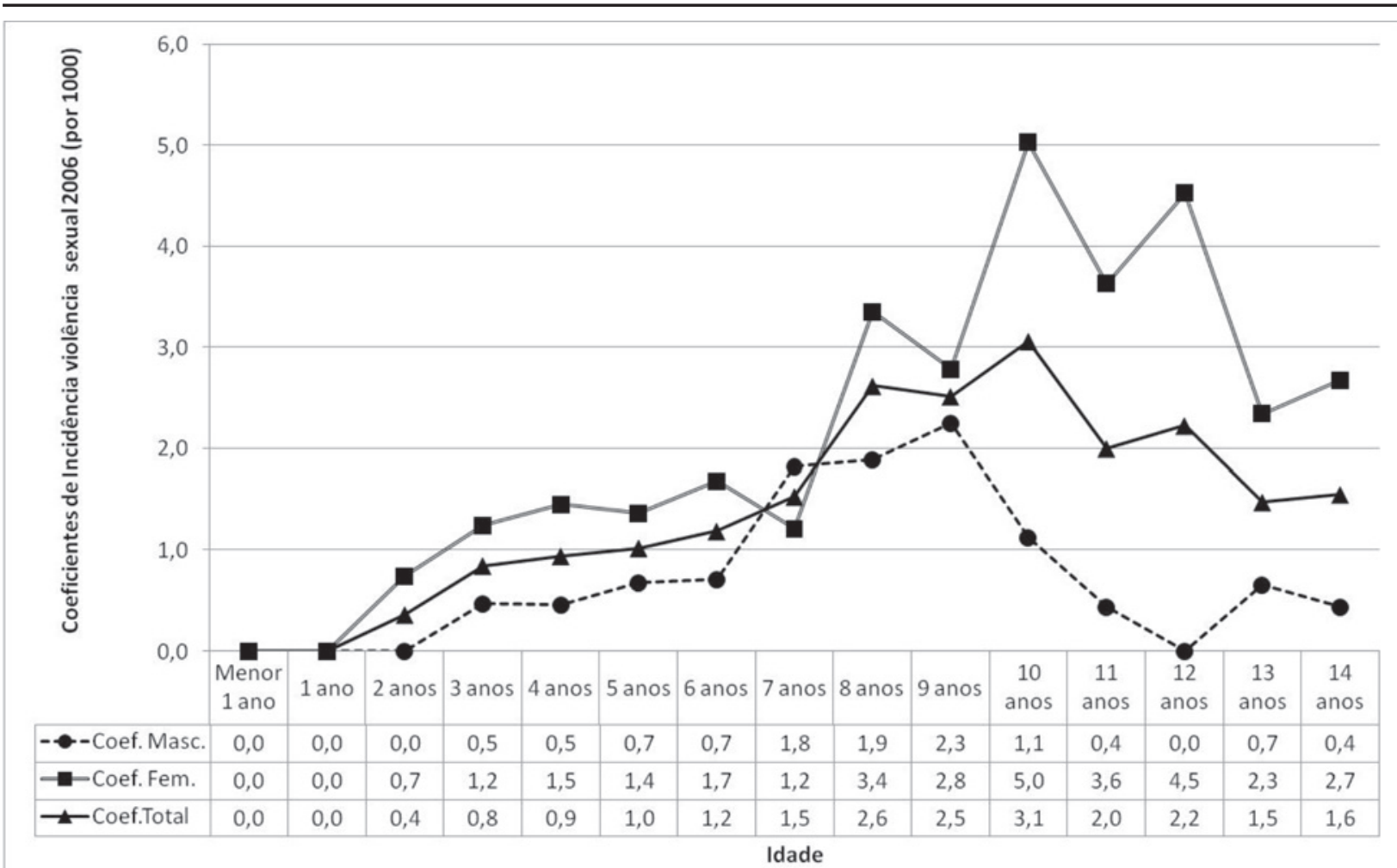

Figura 1 - Coeficientes de incidência de abuso sexual contra crianças e adolescentes de zero a 14 anos, segundo o sexo e a idade das vítimas. Londrina-PR, 2006

O abuso sexual, notificado na presente investigação, foi perpetrado, em sua grande parte, por agressores do sexo masculino $(97,3 \%)$, porém observou-se a presença de mulheres entre os agressores $(2,1 \%)$. A faixa etária com maior freqüência, entre os agressores homens, foi a de
40 anos e mais $(25,4 \%)$ e de 30 a 34 anos $(21,5 \%)$. Verificou-se que em $9,9 \%$ dos casos, adolescentes de 13 a 14 anos foram os agressores de crianças. A idade das mulheres agressoras foi de 20 a 24 $\operatorname{anos}(50,0 \%), 35$ a 39 anos $(25,0 \%)$ e 40 e mais anos $(25,0 \%)$ (Tabela 1$)$.

Tabela 1 - Distribuição dos casos de abuso sexual contra crianças e adolescentes de zero a 14 anos, segundo o sexo e a idade do agressor. Londrina-PR, 2006

\begin{tabular}{lrrrrrr}
\hline \multirow{2}{*}{$\begin{array}{l}\text { Idade do agressor de abuso } \\
\text { sexual }\end{array}$} & \multicolumn{3}{c}{ Mexo do agressor } & \multicolumn{3}{c}{ Total } \\
\cline { 2 - 7 } & $\mathrm{n}$ & $\%$ & $\mathrm{n}$ & $\%$ & $\mathrm{n}$ & $\%$ \\
\hline$<15$ anos & 18 & 9,9 & - & - & 18 & 9,7 \\
15 a 19 anos & 21 & 11,6 & - & - & 21 & 11,3 \\
20 a 24 anos & 10 & 5,5 & 2 & 50,0 & 12 & 6,4 \\
25 a 29 anos & 9 & 5,0 & - & - & 9 & 4,8 \\
30 a 34 anos & 39 & 21,5 & - & - & 39 & 21,0 \\
35 a 39 anos & 33 & 18,2 & 1 & 25,0 & 34 & 18,3 \\
40 e mais anos & 46 & 25,4 & 1 & 25,0 & 47 & 25,3 \\
Ignorada & 4 & 2,2 & - & - & 4 & 2,7 \\
Não informada & 1 & 0,6 & - & - & 1 & 0,5 \\
\hline Total & $\mathbf{1 8 1}$ & $\mathbf{1 0 0 , 0}$ & $\mathbf{4}$ & $\mathbf{1 0 0 , 0}$ & $\mathbf{1 8 5}$ & $\mathbf{1 0 0 , 0}$ \\
\hline
\end{tabular}

* Em um caso o sexo do agressor era ignorado. 
Quanto ao vínculo entre agressores e vítimas, as maiores freqüências se deram nos casos em que os agressores foram o padrasto $(30,1 \%)$, outros parentes como tios, cunhados e primos $(21,5 \%)$, além de vizinhos (18,3\%). Em seguida, vieram os desconhecidos, amigos da família e o próprio pai (Tabela 2).

Tabela 2 - Distribuição dos casos de abuso sexual contra crianças e adolescentes de zero a 14 anos, segundo o vínculo entre agressores e vítimas. Londrina-PR, 2006

\begin{tabular}{lrr}
\hline $\begin{array}{l}\text { Vínculo entre agressor e vítima de } \\
\text { abuso sexual }\end{array}$ & $\mathbf{n}$ & \multicolumn{1}{c}{$\%$} \\
\hline Padrasto & 56 & 30,1 \\
Outros parentes & 40 & 21,5 \\
Vizinhos & 34 & 18,3 \\
Amigo(a) da família & 16 & 8,6 \\
Pai & 13 & 7,0 \\
Colega de escola & 9 & 4,8 \\
Desconhecido & 7 & 3,8 \\
Namorado da mãe & 2 & 1,1 \\
Porteiro do prédio & 2 & 1,1 \\
Vendedor de lanche da escola & 2 & 1,1 \\
Mãe & 1 & 0,5 \\
Babá & 1 & 0,5 \\
Caseiro da chácara & 1 & 0,5 \\
Não informado & 2 & 1,1 \\
\hline Total & $\mathbf{1 8 6}$ & $\mathbf{1 0 0 , 0}$ \\
\hline
\end{tabular}

Um elevado número de prontuários $(66,1 \%)$ não trazia a situação de vulnerabilidade associada ao agressor (alcoolismo, drogadição, desemprego, etc.). Ainda assim, o alcoolismo foi registrado como uma situação freqüente $(24,7 \%)$, seguido pela drogadição $(4,3 \%)$, desemprego $(4,3 \%)$ e distúrbio psiquiátrico $(0,5 \%)$.

A maioria dos casos de abuso sexual ocorreu na residência da vítima $(52,7 \%)$, seguida pela residência do agressor $(30,1 \%)$. Observou-se que mais da metade das vítimas sofreu o abuso sexual pela primeira vez $(52,1 \%)$. Entretanto, houve casos em que a violência sexual havia sido cometida por quatro ou mais vezes (36,6\%). A duração do abuso foi menos de seis meses em $57,0 \%$ dos casos. Uma pequena parcela $(29,0 \%)$ sofreu a violência sexual por um período de um a dois anos antes que o caso fosse notificado (Tabela 3).

Quase todos os casos de violência sexual $(90,3 \%)$ ocasionaram lesão corporal. Nos demais, não foi possível determinar a presença de lesão em decorrência da qualidade da informação. Na gran- de maioria dos casos, o segmento corpóreo mais afetado foi a pelve $(93,1 \%)$, seguida pelos casos em que foram afetadas múltiplas regiões $(6,9 \%)$.

Tabela 3 - Distribuição dos casos de abuso sexual contra crianças e adolescentes de zero a 14 anos, segundo o local, a frequiência e duração do abuso. Londrina-PR, 2006. ( $N=186)$

\begin{tabular}{lcr}
\hline Variáveis & $\mathbf{n}$ & $\%$ \\
\hline Local em que ocorreu & & \\
Residência da vítima & 98 & 52,7 \\
Residência do agressor & 56 & 30,1 \\
Residência de parente & 10 & 5,4 \\
Via pública & 8 & 4,3 \\
Escola & 4 & 2,4 \\
Terreno baldio & 2 & 0,8 \\
Outro & 7 & 3,8 \\
$\quad$ Não informado & 1 & 0,5 \\
Frequência & & \\
Uma vez & 97 & 52,1 \\
4 ou mais vezes & 68 & 36,6 \\
3 vezes & 11 & 5,9 \\
2 vezes & 4 & 2,1 \\
Não informado & 6 & 3,3 \\
Duração & & \\
Menos de 6 meses & 106 & 57,0 \\
1 a 2 anos & 54 & 29,0 \\
6 a 12 meses & 14 & 7,5 \\
3 a 4 anos & 1 & 0,5 \\
Mais de 4 anos & 1 & 0,5 \\
Ignorado & 4 & 2,2 \\
Não informado & 6 & 3,3 \\
\hline
\end{tabular}

Mais de 70,0\% dos prontuários não traziam a informação se houve necessidade de internação. Apenas uma pequena parcela $(11,3 \%)$ tinha o registro de que as vítimas foram internadas em serviço hospitalar.

A maioria das vítimas de abuso sexual apresentou seqüelas $(97,8 \%)$, sendo que a mais comum foi a física $(92,9 \%)$ acompanhada pela seqüela psicológica.

\section{DISCUSSÃO}

Faz-se oportuno ressaltar que, pelo estudo ter se restringido aos casos notificados aos Conselhos Tutelares e programas e projetos de atendimento, certamente os resultados subestimam a real incidência destes eventos na população. Entretanto, acredita-se que esta limitação não seja suficientemente importante para comprometer a interpretação dos resultados apresentados. 
Assim como o presente estudo, em que os familiares foram os principais denunciantes, levantamento realizado em Fortaleza, verificou que a maior parte das denúncias de violência sexual contra crianças e adolescentes foi feita por familiares $(52,0 \%) .{ }^{23}$ Estudo realizado na Bolívia também revela que as mães foram as que mais denunciaram a agressão. ${ }^{24}$

De difícil notificação por envolver tabus e discriminação das vítimas como culpadas, ${ }^{19}$ vários autores consideram a violência sexual subestimada em decorrência do sub-registro, ${ }^{2}$ destacando que os fatores que contribuem para a pouca denúncia das vitimizações sexuais envolvem medo, falta de credibilidade no sistema legal e o silêncio do cúmplice. ${ }^{5}$

Apesar de a própria vítima ter notificado alguns casos, sua baixa freqüência como denunciante é discutida pelos autores como conseqüência do profundo pacto de silêncio que envolve a agressão sexual e a falta de estrutura psicológica da criança para comunicar o fato. Destaca-se, ainda, a culpabilização da criança ou adolescente por ter sido abusada. Um exemplo é a pesquisa realizada na Bolívia, na cidade de El Alto, em que se verificou a violência sexual praticada contra adolescentes menores de 18 anos, ${ }^{24}$ observando que $30,0 \%$ das vítimas, ao relatarem o abuso, foram agredidas fisicamente e responsabilizadas pelo ocorrido. Associadas a estes fatores, a pouca credibilidade no relato da criança ou adolescente e a complicada confirmação da violência fazem com que a vítima dependa, muitas vezes, da iniciativa de outras pessoas fora do núcleo familiar para que a notificação seja feita, ${ }^{2,5,17}$ o que pode explicar a maior proporção de denúncias por familiares e diretores de escola, no presente estudo.

Estudiosos da área ainda discutem o papel de conivência que a mãe assume diante da violência sexual dentro de casa. Para manter a estabilidade e a segurança, a mãe passa a corroborar com a prática do abuso não reconhecendo o incesto, pois revelá-lo representaria reconhecer o fracasso de seu papel como mãe e esposa. Somando-se a isto, ainda se acrescentam o temor da mãe de que ao denunciar o abuso haja rompimento com os parentes e conhecidos, além da incerteza em relação ao futuro ao ter que enfrentar as exigências da vida sem o marido, o trauma de ter que lidar com a polícia e tribunais e a própria incapacidade de opôr-se a uma violência que ela mesma tenha sofrido. ${ }^{24}$ Talvez estas questões justifiquem a tímida participação da mãe como notificadora, na presente investigação.
O predomínio do sexo feminino na violência sexual também é reconhecido por autores nacionais e internacionais. ${ }^{12,16,24}$ Pesquisa realizada junto aos registros do Centro Regional de Atenção aos Maus-Tratos na Infância de São José do Rio PretoSP, observou uma razão de masculinidade de 1:7, entre as vítimas de violência sexual. ${ }^{3}$ A maior proporção de vítimas do sexo feminino pode ser explicada pelas próprias questões de gênero, que ainda colocam a mulher como um objeto sexual, apesar das conquistas femininas atuais, ${ }^{16}$ fazendo com que crianças e adolescentes meninas sejam os alvos preferenciais dos agressores. ${ }^{5}$

Quanto à faixa etária, estudo sobre violência sexual, realizado na Região Metropolitana de Fortaleza, a partir de notificações dos Conselhos Tutelares e do Sistema de Informação para a Infância e Adolescência (SIPIA), também verificou proporção elevada de vítimas na faixa etária de 10 a 14 anos, com uma média de idade de 11,4 anos. ${ }^{23}$ Em levantamento junto ao Organismo Nacional de La Mujery Familia (ONAMFA), na cidade de El Alto - Bolívia, o grupo etário de 10 a 14 anos representou 44,1\% das vítimas de abuso sexual, ${ }^{24}$ com predomínio da idade de cinco anos entre as crianças do sexo masculino. Tais resultados assemelham-se à presente investigação. Outra casuística realizada na Venezuela encontrou, entre vítimas de agressão sexual, maior incidência de adolescentes de 12 a 18 anos (43,7\%); escolares de sete a 11 anos $(22,8 \%)$ e pré-escolares de três a seis anos $(17,2 \%)$, representando um total de $83,8 \%{ }^{12}$

O predomínio de casos femininos na faixa etária de 10 a 14 anos revela a preferência dos agressores por meninas na puberdade, quando se desenvolvem os caracteres sexuais secundários. Ao contrário, entre os meninos, a pouca idade em que são vítimas com mais freqüência pode estar relacionada com a impossibilidade de defesa, o que não ocorre em idades mais avançadas.

Há estimativas de que $36,0 \%$ das meninas e $29,0 \%$ dos meninos, no mundo todo, sofram abuso sexual e, pelo menos, uma em cada cinco mulheres tenham sofrido abuso sexual em algum momento de sua vida. ${ }^{10}$ Entretanto, é preciso ressaltar que menos de 10,0\% dos casos chegam às delegacias (campo de estudo do referido trabalho) pelo temor que as vítimas têm em relação às medidas legais de confirmação do abuso e à exposição social. ${ }^{5}$ Em São Paulo, dos 52.965 casos de violência doméstica contra crianças e adolescentes, atendidas pelo LACRI (Laboratório de Estudos da Criança) do Instituto de Psicologia da USP (IPUSP), no período de 1996 
a $2001,8,0 \%$ foram por abuso sexual. ${ }^{15}$ Em Curitiba, a violência sexual representou 6,2\% das 2.326 notificações emitidas pelos serviços que compõem a Rede de Proteção à Criança e ao Adolescente em Situação de Risco para a Violência. ${ }^{16}$

Pesquisa domiciliar realizada na Carolina do Norte e do Sul evidenciou o abuso sexual entre as crianças de mães entrevistadas, resultando numa incidência de 23 casos por 1.000 crianças (29 por 1.000 entre as meninas e três por 1.000 entre os meninos), proporções elevadas se comparadas à presente investigação. ${ }^{4}$ Porém, há que se considerar que se trata de entrevista domiciliar, cujos casos nem sempre são reportados aos órgãos oficiais. Nos Estados Unidos, a incidência de abuso sexual já chegou a 2,6 por 1.000 adolescentes na faixa etária de 12 a 14 anos e de 2,9 por 1.000 adolescentes de 15 a 19 anos de idade, ${ }^{14}$ taxas mais altas que as encontradas no presente estudo, o que pode estar relacionado com a melhor qualidade da informação e da notificação naquele país.

Estatísticas apontam que na Costa Rica, 3,2\% das meninas e $13,0 \%$ dos meninos são abusados sexualmente ao ano. Na Nicarágua, estes percentuais são de $26,0 \%$ para as meninas e $20,0 \%$ para os meninos. ${ }^{25}$

A Associação Brasileira de Proteção à Infância e Adolescência (ABRAPIA), no Rio de Janeiro, realizou 3.981 atendimentos a crianças vitimizadas no lar no período de 1991 a 1993. ${ }^{26}$

Apesar dos estudos que se esforçam para delinear uma incidência próxima da realidade, muitos autores nacionais e internacionais ainda consideram a violência sexual de prevalência desconhecida, uma vez que, na maioria das vezes, a criança só consegue revelar o abuso na fase adulta, além do silencio e tabus que envolvem este tipo de agressão e da dificuldade de alguns países em desenvolver um sistema de vigilância e de informação adequados. ${ }^{5,8,27}$

A presente investigação coincide com estudos realizados anteriormente sobre violência sexual, que apontam o sexo masculino predominante entre os agressores. ${ }^{3} \mathrm{O}$ predomínio de agressores sexuais do sexo masculino reforça a discussão de gênero acerca da exploração e dominação dos homens sobre o sexo feminino, especialmente sobre as crianças, perpetuando o padrão cultural da sociedade patriarcal. ${ }^{12,28}$ Neste sentido, autores destacam que as diferenças entre os sexos se convertem em desigualdades que possibilitam o processo de dominação e exploração. ${ }^{5}$

Na presente casuística, a maior concentração de agressores na faixa etária de 30 a 40 anos, entre os homens, provavelmente se relaciona, entre muitos fatores, com o fato de que nestas idades muitos homens podem já ter constituído uma segunda família, na qual a convivência com enteadas pode facilitar o abuso, tendo em vista que grande parte dos agressores são os padrastos. ${ }^{1,24}$ Entretanto, poucos são os trabalhos que caracterizam o agressor de violência sexual segundo sua idade.

A participação de adolescentes entre os agressores, verificada em outra casuística, desperta para a reflexão acerca desta fase, em que as descobertas e incertezas quanto à sexualidade assumem proporções importantes no comportamento do jovem. ${ }^{29}$ Neste sentido, autores destacam a importância de programas voltados para a adolescência, nos quais se aborde as questões da sexualidade e da vulnerabilidade a que estes jovens estão expostos. ${ }^{29}$

A participação do padrasto, outros parentes, vizinhos, pais e amigos da família, como agressores nos casos de abuso sexual, é amplamente divulgada pelas pesquisas, desde as mais antigas até as mais atuais, ${ }^{12,19}$ revelando que o agressor sexual é, na maioria das vezes, alguém próximo à criança. Além da proximidade, que facilita a abordagem, estas pessoas contam com a confiança das vítimas, usando a relação de confiança para se aproximar cada vez mais, num processo insidioso, sem que a criança perceba o ato abusivo do adulto.

A presença do alcoolismo como situação associada ao abuso também foi verificada em outra pesquisa, que identificou tal circunstância presente em 35,0\% das famílias bolivianas, cujas crianças foram abusadas sexualmente. ${ }^{24}$ Vários autores apontam o alcoolismo como variável possivelmente relacionada com a ocorrência de maus-tratos contra crianças e adolescentes, ${ }^{3,19}$ o que chama a atenção para a necessidade de campanhas e programas públicos de fácil acesso que visem à prevenção e o tratamento adequado dos dependentes.

Outras pesquisas apontam o ambiente doméstico como o local onde mais ocorre a violência sexual. ${ }^{5}$ Estudo realizado na Bolívia, na cidade de El Alto, observou que mais da metade $(54,7 \%)$ dos casos de violência sexual contra menores de 18 anos ocorreram no domicílio da vítima. ${ }^{24} \mathrm{~A}$ maior proporção de agressão sexual cometida dentro de casa suscita a reflexão de que o lar, muitas vezes, não constitui um local seguro para as crianças, uma vez que a agressão pode ser praticada sem que a sociedade tenha conhecimento.

Apesar da metade das vítimas de violência sexual ter sofrido a agressão pela primeira vez e por menos de seis meses, a presença do abuso por qua- 
tro ou mais vezes e por um período de um a dois anos, caracteriza o aspecto insidioso da violência sexual contra crianças e adolescentes, ${ }^{2}$ em que estes não conseguem verbalizar o abuso para terceiros, em decorrência do poder e persuasão do agressor para manter o abuso em segredo. Autores apontam que o longo período de abuso está relacionado com o suborno e a ameaça para assegurar o silêncio de suas vítimas. ${ }^{24}$ Além destas características que envolvem a agressão sexual, ainda se destaca o fato de que, dificilmente, os agressores sexuais procuram tratamento voluntariamente, pois há um manto de silêncio e a reprovação moral que envolve a agressão sexual, ${ }^{2}$ sem o qual a agressão tende a continuar, contribuindo para que a criança sofra o abuso por várias vezes e longos períodos.

Os resultados encontrados quanto à lesão, em que o segmento corpóreo mais afetado foi a pelve $(93,1 \%)$, coincide com outras pesquisas que apontam para a importância das lesões decorrentes da agressão sexual. Além das lesões genitais e anais, investigação entre crianças e adolescentes bolivianas vítimas de abuso sexual, encontrou a presença de doenças sexualmente transmissíveis (infecções gonocócicas e sífilis), lesões por mordedura, alopécia por arrancamento dos cabelos, além de fratura óssea em úmero e clavícula. ${ }^{24}$ Estes resultados alarmantes trazem consigo o agravo de que muitas lesões podem trazer conseqüências para a vida reprodutiva destas vítimas, além da possibilidade de gravidez decorrente do estupro, situação verificada em 10 das 140 denúncias em estudo realizado. ${ }^{24}$ Considerando a gravidade das lesões apontada por esses autores, torna-se essencial atenção especializada e continuada à saúde física e emocional destas vítimas, além de acompanhamento àquelas que engravidaram em conseqüência do abuso.

Justifica-se a pelve ter sido mais afetada justamente pelas lesões genitais decorrentes do abuso sexual. Já o acometimento de múltiplas regiões pode ter sido em decorrência de violência física associada.

Durante o levantamento bibliográfico, não foram encontrados estudos acerca da violência sexual que analisassem a necessidade de internação em suas vítimas. Todavia, tendo em vista as lesões apontadas nos estudos supracitados, em conseqüência da violência sexual, é possível que grande parte dos casos necessite de internação, embora não haja relato deste dado.

Além das lesões físicas e genitais sofridas, a possibilidade de uma doença sexualmente transmissível e de uma gravidez indesejada constitui uma triste realidade, ${ }^{5,19}$ como discutido anteriormente. Mais grave, ainda, são as seqüelas psicológicas, relatadas em todas as vítimas da presente investigação.

Neste aspecto, os autores ressaltam o grande impacto que a agressão sexual exerce na saúde física e mental das vítimas, deixando marcas profundas e causando danos irreparáveis em seu desenvolvimento, com impacto na vida reprodutiva e sexual. ${ }^{24}$ Em curto prazo, a experiência do abuso sexual pode ocasionar em suas vítimas distúrbios de alimentação e do sono, descontrole esfincteriano, problemas escolares, interesse sexual e atitudes provocativas precoces, depressão, fuga de casa, tentativa de suicídio, uso de drogas, ansiedade e agressividade. Vale ressaltar que, apesar de algumas notificações não constarem a seqüela psicológica imediata, essas crianças e adolescentes poderão desenvolver problemas psicológicos e distúrbios psiquiátricos em longo prazo. Ainda, a agressão sexual pode levar à exploração e delinqüência na adolescência, a distúrbios psicossomáticos, desvios e transtornos sexuais, tais como frigidez, vaginismo, impotência, homossexualismo masculino e feminino, troca freqüente de parceiro, entre outros. ${ }^{19}$

\section{CONCLUSÃO}

Diante dos danos e agravos que a violência sexual determina entre crianças e adolescentes, este tipo de abuso constitui-se um problema de saúde pública, além da estreita interface com as questões policiais e jurídicas. Há que se considerar, todavia, que somente medidas punitivas não solucionam um problema tão complexo quanto a agressão sexual. Desta forma, é preciso que os órgãos competentes e os diversos setores (conselhos tutelares, poder judiciário, instituições de saúde, escolas, creches, instituições de atendimento a crianças e adolescentes, organizações governamentais e não governamentais) trabalhem para a detecção precoce, a fim de interromper sua ocorrência e possibilitar tratamento e acompanhamento adequados que possam reduzir as importantes e devastadoras seqüelas decorrentes.

Por constituir-se um grande desafio aos diversos setores e profissionais que se deparam com sua ocorrência, o fenômeno da violência sexual demanda investimentos na capacitação dos profissionais que lidam, direta ou indiretamente, com crianças e adolescentes, pois a dificuldade na identificação da violência sexual, muitas vezes, está relacionada ao desconhecimento destes profissionais. Há que se destacar a necessidade destes 
profissionais se apropriarem de conhecimentos acerca das penalidades aplicáveis ao profissional que não denuncia a suspeita ou a ocorrência da violência conforme o Estatuto da Criança e do Adolescente, sobre a identificação e as conseqüências da violência sexual para o desenvolvimento infanto-juvenil. É preciso que os profissionais de saúde e educação, principalmente, estejam atentos para os sinais e sintomas do abuso sexual entre crianças e adolescentes, visando à detecção e tratamento adequados. Além disto, o conhecimento da legislação e dos meios de proteção legal torna-se necessário na assistência às vítimas.

Além da identificação, essencial para o conhecimento das características e magnitude do agravo, torna-se essencial refletir sobre o atendimento, tratamento e acompanhamento das vítimas e famílias. É preciso estruturar os serviços de saúde para que se possa oferecer atendimento terapêutico adequado, numa ampla rede de apoio, a fim de que se possa minimizar as importantes seqüelas da violência sexual.

Destaca-se, ainda, a importância de ações educativas preventivas em todos os níveis de atenção, além da promoção dos fatores de proteção, o que pode contribuir para a redução da violência sexual e seu conseqüente impacto sobre o desenvolvimento da criança e adolescente.

A presente pesquisa aponta para a relevância e necessidade de estudos científicos que permitam conhecer melhor o evento, incluindo sua relação com os fatores predisponentes, o que pode direcionar melhor as ações de prevenção e os serviços de atendimento.

Ressalta-se, oportunamente, a importância do preenchimento completo e correto dos prontuários no momento do atendimento, o que pode contribuir para a qualidade da notificação e conseqüente melhora na visibilidade do fenômeno.

\section{AGRADECIMENTOS}

Agradecemos à Coordenação de Aperfeiçoamento de Pessoal de Nível Superior - CAPES e ao Conselho Nacional de Desenvolvimento Científico e Tecnológico - CNPq pela concessão de bolsas de estudo.

\section{REFERÊNCIAS}

1. Peláez Mendoza J, Juncal HV. Abuso sexual en niñas y adolescentes: experiencias de 10 años. Rev Cubana Obstet Ginecol. 2009 Mar; 35(1):1-13.
2. Morales AE, Schramm FR. A moralidade do abuso sexual intrafamiliar em menores. Cienc Saude Colet. 2002 Fev; 7(2):265-73.

3. Brito AM, Zanetta DM, Mendonça RC, Barison SZ, Andrade VAG. Violência doméstica contra crianças e adolescentes: estudo de um programa de intervenção. Cienc Saude Colet. 2005 Set; 10(1):143-9.

4. Theodore AD, Chang JJ, Runyan DK, Hunter WM, Bangdiwala SI, Agans R. Epidemiologic features of the physical and sexual maltreatment of children in the Carolinas. Pediatrics. 2005 Mar; 115(3):331-7.

5. Ribeiro MA, Ferriani MGC, Reis JN. Violência sexual contra crianças e adolescentes: características relativas à vitimização nas relações familiares. Cad Saúde Pública. 2004 Mar-Abr; 20(2):456-64.

6. Ortega MAF, Terrés BEV, Bannack MED, Quintanilha EG, Ramírez MPP, Ortega VS, et al. La experiência de um programa de atencíon a la violência em uma unidad de primer nível para población abierta. Rev Fac Med UNAM. 2001 Ago; 44(2):49-54.

7. Faria TD, Oliveira PAF, Mendes R. O enfrentamento à exploração sexual de crianças e adolescentes: desafios e caminhos. Cienc Saude Colet. 2007 JulSet; 12(5):115-8.

8. Ministério da Saúde (BR), Secretaria de Políticas de Saúde. Prevenção e tratamento dos agravos resultantes da violência sexual contra mulheres e adolescentes: normas técnicas. Brasília (DF): MS; 1999.

9. Ministério da Justiça (BR), Secretaria Estadual de Direitos Humanos. Plano Nacional de enfrentamento da violência sexual infanto-juvenil. Brasília (DF): MJ; 2001.

10. Organización Panamericana de la Salud (OMS). Repercusión de la violencia en la salud de las poblaciones americanas. Washington (US): OMS; 2003.

11. Organização Pan-Americana de Saúde [página na Internet]. Portal da OPAS; 2003. [acesso 2010 Mar. 18]. Disponível em: www.opas.org.br

12. Nunez A, Tortolero Y, Verschuur A, Camacaro M, Mendoza S. Violencia sexual: un fenómeno oculto en la experticia médico legal. Rev Obstet Ginecol Venez. 2008 Dez; 68(4):233-9.

13. Creighton SJ. Prevalence and incidence of child abuse: international comparisons. NSPCC Information Briefings. 2004. [acesso 2010 Mar 18]. Disponível em: www.nspcc.org.uk/Inform/OnlineResources/ InformationBriefings/PrevalenceAndIncidenceOfChildAbuse_asp_ifega26028html.

14. Cappelleri JC, Eckenrode J, Powers JL. The epidemiology of child abuse: findings from the second national incidence and prevalence study of child abuse and neglect. Am J Public Health. 1993 Nov; 83(11):1622-4.

15. Laboratório de Estudos da Criança - LACRI [página na Internet]. São Paulo (SP): Instituto de Psicologia da 
Universidade de São Paulo; 2008 [acesso 2010 Mar 18]. Disponível em: www.ip.usp.br/laboratorios/lacri

16. Oliveira VLA. A violência doméstica contra crianças e adolescentes no Município de Curitiba [dissertação]. São Paulo (SP): Universidade de São Paulo. Programa de Pós-Graduação em Saúde Pública; 2006.

17. Machado HB, Lueneberg CF, Régis E, Nunes MPP. Abuso sexual: diagnóstico de casos notificados no município de Itajaí-SC, no período de 1999 a 2003, como instrumento para a intervenção com famílias que vivenciam situações de violência. Texto Contexto Enferm. 2005; 14(Esp):54-63.

18. Roque EMST, Ferriani MGC. Desvendando a violência doméstica contra crianças e adolescentes sob a ótica dos operadores do direito na Comarca de Jardinópolis - SP. Rev Latino-am Enfermagem. 2002 Mai-Jun; 10(3):334-44.

19. Minayo MCS. Violência contra crianças e adolescentes: questão social, questão de saúde. Rev Bras Saúde Mater-infant. 2001 Jan-Abr: 1(1):91-102.

20. Secretaria Municipal de Saúde de Londrina. Boletim Informativo da Saúde [online]; 2003 [acesso 2010 Mar 18]; VII(35):4-6. Disponível em: http//:www. londrina.pr.gov.br

21. Suguihiro VLT, Barros MNF. A importância da Ação Interdisciplinar para uma política de inclusão social. Anais do Seminário Latino-Americano de Serviço Social [CD-ROM]. Florianópolis (SC): ABEPSS; 2004. p.25.

22. Dean AG, Dean JA, Coulombier D, Brendel KA, Smith DC, Burten AH, et al. EPI INFO version 6: word processing, database and statistics program for epidemiology on microcomputers. Atlanta (US): Centers for Disease Control and Prevention; 1995.
23. Lins MZS. Avaliação do sistema de informação para a infância e adolescência (SIPIA), com foco na notificação de violência sexual na Região Metropolitana de Fortaleza, no período de 1999 a 2007 [tese]. Rio de Janeiro (RJ): Escola Nacional de Saúde Pública Sergio Arouca. Programa de PósGraduação em Saúde Pública; 2008.

24. Guerra RDO. Abuso sexual em ninas y ninos - consideraciones clínicas. J Pediatr. 2000 Mar; 76(4):C33-8.

25. World Health Organization - WHO [página na Internet]. Preventing Violence - a guide to implementing the recommendations of the World Report on Violence and Health. Geneva: WHO; 2004. [acesso 2010 Mar 18]. Disponível em: www.who.int/gb

26. Associação Brasileira de Proteção à Infância e à Adolescência - ABRAPIA [página na Internet]. São Paulo (SP): ABRAPIA; 2010 [acesso 2010 Mar 18]. Disponível em: www.abrapia.org.br

27. Heger A, Ticson L, Velasquez O, Bernier R. Children referred for possible sexual abuse: medical findings in 2384 children. Child Abuse Negl. 2002 Jun; 26(4):645-59.

28. Chavez AR, Rivera-Rivera L, Angeles-Llerenas A, Díaz-Cerón E, Allen-Leigh B, Lazcano PE. Factores del abuso sexual en la niñez y la adolescencia en estudiantes de Morelos, México. Rev Saúde Pública. 2009 Mai-Jun; 43(3):506-514.

29. Monteiro CFS, Teles DCBS, Castro KL, Vasconcelos NSV, Magalhães RLB, Deus MCBR. Violência sexual contra criança no meio intrafamiliar atendidos no SAMVVIS, Teresina, PI. Rev Bras Enferm. 2008 JulAgo; 61(4):459-63. 